\title{
Video Article \\ Combined Intravital Microscopy and Contrast-enhanced Ultrasonography of the Mouse Hindlimb to Study Insulin-induced Vasodilation and Muscle Perfusion
}

\author{
Alexander H. Turaihi ${ }^{1}$, Erik M. van Poelgeest ${ }^{1}$, Victor W. M. van Hinsbergh ${ }^{1}$, Erik H. Serné ${ }^{2}$, Yvo M. Smulders ${ }^{2}$, Etto C. Eringa ${ }^{1}$ \\ ${ }^{1}$ Laboratory for Physiology, Institute for Cardiovascular Research (ICaR-VU), VU University Medical Center \\ ${ }^{2}$ Department of Internal Medicine, Institute for Cardiovascular Research (ICaR-VU), VU University Medical Center
}

Correspondence to: Alexander H. Turaihi at a.turaihi@vumc.nl

URL: https://www.jove.com/video/54912

DOI: doi:10.3791/54912

Keywords: Medicine, Issue 121, Intravital microscopy, contrast-enhanced ultrasonography, insulin sensitivity, vascular responsiveness, endothelium, microsurgery

Date Published: 3/20/2017

Citation: Turaihi, A.H., van Poelgeest, E.M., van Hinsbergh, V.W., Serné, E.H., Smulders, Y.M., Eringa, E.C. Combined Intravital Microscopy and Contrast-enhanced Ultrasonography of the Mouse Hindlimb to Study Insulin-induced Vasodilation and Muscle Perfusion. J. Vis. Exp. (121), e54912, doi:10.3791/54912 (2017).

\section{Abstract}

It has been demonstrated that insulin's vascular actions contribute to regulation of insulin sensitivity. Insulin's effects on muscle perfusion regulate postprandial delivery of nutrients and hormones to insulin-sensitive tissues. We here describe a technique for combining intravital microscopy (IVM) and contrast-enhanced ultrasonography (CEUS) of the adductor compartment of the mouse hindlimb to simultaneously visualize muscle resistance arteries and perfusion of the microcirculation in vivo. Simultaneously assessing insulin's effect at multiple levels of the vascular tree is important to study relationships between insulin's multiple vasoactive effects and muscle perfusion. Experiments in this study were performed in mice. First, the tail vein cannula is inserted for the infusion of anesthesia, vasoactive compounds and ultrasound contrast agent (lipid-encapsulated microbubbles). Second, a small incision is made in the groin area to expose the arterial tree of the adductor muscle compartment. The ultrasound probe is then positioned at the contralateral upper hindlimb to view the muscles in cross-section. To assess baseline parameters, the arterial diameter is assessed and microbubbles are subsequently infused at a constant rate to estimate muscle blood flow and microvascular blood volume (MBV). When applied before and during a hyperinsulinemic-euglycemic clamp, combined IVM and CEUS allow assessment of insulin-induced changes of arterial diameter, microvascular muscle perfusion and whole-body insulin sensitivity. Moreover, the temporal relationship between responses of the microcirculation and the resistance arteries to insulin can be quantified. It is also possible to follow-up the mice longitudinally in time, making it a valuable tool to study changes in vascular and whole-body insulin sensitivity.

\section{Video Link}

The video component of this article can be found at https://www.jove.com/video/54912/

\section{Introduction}

In response to a rise in blood glucose level, the pancreas secretes insulin into the bloodstream where it is quickly distributed to its target tissues such as skeletal muscle, via resistance arteries and capillaries. Skeletal muscle is responsible for $\sim 80 \%$ of postprandial glucose uptake ${ }^{1}$. The delivery of insulin to the skeletal muscle interstitium has been shown to be a rate limiting step for the metabolic actions of insulin that promote glucose disposal ${ }^{2,3,4}$. Within 10-15 min, insulin increases the capillary blood volume (microvascular recruitment), an effect that occurs before total blood flow increases ${ }^{5,6}$. Microvascular recruitment expands the endothelial surface area available for exchange of nutrients (and insulin) ${ }^{7,8}$ Insulin-mediated microvascular recruitment precedes and is independently associated with changes in skeletal muscle glucose uptake ${ }^{8,9}$. The effect of insulin on the vasculature has been termed 'vascular insulin sensitivity'.

It has been shown that insulin-mediated microvascular recruitment and insulin-induced vasodilation are impaired in obese Zucker rats ${ }^{10,11}$ Moreover, lean mice with reduced capillary density display muscle insulin resistance ${ }^{12}$. In their influential work, Kubota et al. showed that impaired insulin signaling in endothelial cells caused reduction in insulin-induced microvascular recruitment, which reduced glucose uptake in skeletal muscle by approximately $40 \%{ }^{13}$. These abnormalities in microvascular function do not only occur in muscle, but also in multiple other tissues and organs such as the heart, retina and kidney ${ }^{14,15,16}$. These examples and other studies ${ }^{17,18,19,20}$ suggest that vascular effects of insulin are an important mechanism in the (patho)physiology of insulin resistance and its complications.

While there is substantial evidence that insulin increases microvascular blood volume (MBV) in skeletal muscle ${ }^{5,6}$, the mechanisms by which this happens are not completely understood ${ }^{9}$. Endothelium-dependent vasodilation is essential in many aspects of vascular insulin sensitivity ${ }^{21,22,23}$ at different levels of the vasculature. Vascular insulin sensitivity can manifest itself by insulin-induced relaxation of resistance arteries and by relaxation of pre-capillary arterioles to increase the perfused microvascular exchange surface area ${ }^{7,24,25}$. 
Intravital microscopy (IVM) has been used in a variety of tissue preparations including skinfold chambers of the mouse dorsum ${ }^{26}$, the mesentery of the mouse and rat $^{27}$, models of limb ischemia in the mouse ${ }^{28}$ and the hamster cheek pouch ${ }^{29}$. Contrast-enhanced ultrasonography (CEUS) is another imaging technique that allows assessment of the microcirculation in cardiac ${ }^{30}$ as well as skeletal muscle ${ }^{31}$. It utilizes inert gas-filled microbubbles which behave rheologically as red blood cells and remain entirely within the vascular lumen. These microbubbles are infused intravenously at a constant rate to attain a steady state. A high energy ultrasound wave, then, can be used to destroy the microbubbles. The microbubbles' replenishment speed in the region of interest (ROI) represents flow velocity (MFV). The total signal intensity of the contrast image represents the MBV. CEUS can be performed repeatedly (also in humans) and it has advanced the understanding of vascular dysfunction that occurs in insulin-resistant states (discussed in Barrett et al. ${ }^{32}$ ).

In the current study we describe a new technique for studying regulation of muscle perfusion, through simultaneous use of both IVM and CEUS. Here we focus on insulin's vascular actions in the adductor compartment of the mouse hindlimb. This compartment is one of the largest skeletal muscle groups in the mouse, enabling studies of local glucose uptake in a representative muscle. This compartment is ideal for IVM as the preparation and visualization of the arteries are readily accessible by a standardized surgical procedure ${ }^{28}$. Moreover, our own group and others have shown that CEUS can be used in this compartment ${ }^{33,34}$.

An advantage to the combined IVM and CEUS technique is the possibility to assess insulin's effect at the level of the larger arterioles (feed or resistance arteries) and the microcirculation (capillary beds) in the same muscle group. In addition, the simultaneous application of the two methods provides insight into the temporal action of insulin at the levels of the resistance arteries and microcirculation. This combined IVM and CEUS technique can also be implemented in other vascular biology fields. For example, the role of various proteins and certain pathophysiological conditions affecting the endothelium can be studied using knockout models. Moreover, both techniques can be used in one mouse at multiple time points reducing the time and cost of research.

\section{Protocol}

All animal experiments have been approved by the local animal care and ethical committee. The entire protocol from induction of anesthesia in the mouse until the end of the hyperinsulinemic-euglycemic clamp takes approximately $2 \mathrm{~h}$.

\section{Microsurgical Preparation}

1. Induce anesthesia along with analgesia in a male mouse weighing $20-25 \mathrm{~g}$ after a $14 \mathrm{~h}$ or overnight fasting with an intraperitoneal injection of a Fentanyl $(0.31 \mathrm{mg} / \mathrm{kg})$, Midazolam $(6.25 \mathrm{mg} / \mathrm{kg})$ and Acepromazine $(6.25 \mathrm{mg} / \mathrm{kg})(\mathrm{FMA}$ anesthesia) and place it on a rectal-temperaturecontrolled homeothermic heating pad which maintains body temperature at $37^{\circ} \mathrm{C}$.

2. Disinfect the operation table and the equipment several times using an alcohol-based solution.

3. Attach a $27 \mathrm{G}$ needle to a $10 \mathrm{~cm}$ long polyethylene tube (PE-20) and attach the tube to a 4-way connector. Insert the needle in the tail vein and fixate it using tissue adhesive gel. This cannula will be used for infusion of microbubbles, insulin, glucose and anesthesia. Note: Adding Heparin $(5 \mathrm{U} / \mathrm{mL})$ in sterile saline during the cannulation process to flush the tail vein (approximately $10 \mu \mathrm{L}$ ) reduces the possibility of clogged cannulas.

4. Throughout the surgical procedures and experimental protocols, maintain anesthesia by a continuous intravenous infusion of FMA anesthesia via the tail vein cannula at rate of $33.75 \mu \mathrm{L} / \mathrm{kg} / \mathrm{min}$.

5. Place the mouse with the ventral side up and fix the feet using a thermostable tape to expose the upper thigh area. Use a slight exorotation of the hip joint (hindlimb paws facing up) and a $40-60^{\circ}$ angle at the knee joint to standardize the stretch of the muscle at the adductor compartment of the thigh.

6. Remove the hair at the groin and upper thigh areas bilaterally using a depilatory cream. Collect all the loose hair with a moist cotton swab.

7. Place the mouse under a stereomicroscope and perform the following surgical steps using 10X to 16X magnification.

8. Make a $2 \mathrm{~cm}$ incision using skin scissors running parallel to the inguinal ligament, just lateral to the abdominal curvature (Figure 1). Apply traction on the distal side of the incision distally using a bulldog clamp (Figure 1D). This will help to adjust the window as needed and help hold the paraffin oil (described in 1.12).

9. Dissect the adipose tissue away from the abdominal wall. To avoid bleeding, gently separate the fat pad from the wall instead of dissecting directly through the pad. Gentle traction at the fat pad in the distal direction will facilitate the process (Figure 1C).

10. Identify the femoral artery and follow it down to the first major branches (the epigastric artery and the gracilis artery) (Figure 2). The gracilis artery is the first major branch of the femoral artery running on the adductor magnus muscle and then runs deep to the gracilis muscle. The gracilis artery will be used for IVM.

11. Identify the transparent deep fascia covering the muscles and the vessels. Using sharp forceps, pull the fascia upwards and cut it using a microscissor.

12. Cover the exposed muscle with a drop $(200 \mu \mathrm{L})$ of medicinal liquid paraffin (room temperature, or pre-warmed to $37^{\circ} \mathrm{C}$ ) to prevent the prepared tissue from drying out. Make sure that the oil drop does not leak away. Adjust the skin folds of the incision using the bulldog clamp to create a small cavity to hold the paraffin oil that bathes the vessels.

13. Place the mouse under a previously calibrated microscope (16X optical magnification) in such a way that the gracilis artery is vertical on the computer screen. Attach the microscope to a camera and computer based analysis system that can extract the vessels' diameter from the image data set. The diameter is the distance between the two luminal sides of the vessel. Continuous monitoring and measurement of the artery diameter is desirable.

14. Place the light source at a sufficient distance (minimum $20 \mathrm{~cm}$ ) from the hindlimb to reduce head conduction from the light.

15. Apply prewarmed ultrasound transduction gel to the upper contralateral hindlimb. Place the ultrasound probe perpendicular to the long axis of the femur bone.

16. Carefully adjust the angle and direction of the ultrasound probe to get a cross-sectional view of the adductor muscle group. Take care to keep the position of the ultrasound probe in relation to the mouse stable to keep the same imaging plane for baseline and hyperinsulinemic measurements.

17. Let the mouse stabilize for $30 \mathrm{~min}$. The diameter of the gracilis artery should be stable for $10 \mathrm{~min}$ before documenting the baseline diameter 


\section{Baseline and Hyperinsulinemic Measurements}

1. Make sure that the baseline gracilis artery diameter is saved by the computer program used for the IVM.

2. Prepare the microbubbles in advance as described ${ }^{35}$ and count with Coulter counter to a concentration of $2.5 \times 10^{9}$ bubbles $/ \mathrm{mL}$ just before the experiment.

3. As the microbubbles can only be viewed in the contrast-mode of the ultrasound machine, control the parameters which affect the image and the contrast data collected (described in 2.3.1) and use consistently during acquisition.

1. Use the following settings on the ultrasound machine: contrast gain at 35 decibels; the time gain compensation to OFF; Line Density to HIGH; Number of Focal Zones to WIDE; Transmit Power to 4\%; Transmit Beam width to STANDARD; SV Gate to 4; Sensitivity to 1; Persistence to OFF. Level the location of Focal Zones to the center of the region of interest.

4. Save a short $(5 \mathrm{~s})$ clip. This will be used to calculate the background signal.

5. Shake the vial containing the microbubbles by hand to have a uniform suspension. Start infusing the microbubbles using the tail vein cannula at a $5 \mu \mathrm{L} / \mathrm{min}$ rate. Place the infusion tube on a vibrating vortex (200x per min) to maintain a uniform suspension of microbubbles.

6. Allow 5 min of continuous infusion of microbubbles so that a steady-state level is reached. Proceed with obtaining the time-intensity curves of the bubbles using the microbubble destruct function (MBD) on the ultrasound machine at 5 min and at 10 min after the start of microbubbles infusion (Figure 3A). Take the average signal of these two measurements to get the baseline perfusion data.

7. After obtaining the baseline data, start the hyperinsulinemic-euglycemic clamp as described ${ }^{34}$. Use the tail cannula (placed in 1.3 ) to administer the insulin and glucose (and anesthesia).

1. In short, induce a hyperinsulinemic state by introducing a $200 \mathrm{mU} / \mathrm{kg}$ insulin bolus followed by continuous insulin infusion $(7.5 \mathrm{mU} / \mathrm{kg} /$ $\min$ ) for $60 \mathrm{~min}$. Use a variable infusion of $20 \% \mathrm{D}$-glucose to maintain euglycemia.

2. Assess blood glucose from the tail vein every $5 \mathrm{~min}$ with a Glucose Monitoring Device, and maintain at $5 \mathrm{mM}$ by adjusting the variable glucose infusion rate. Determine insulin sensitivity by averaging the mean glucose infusion rate during the last 30 min.

8. Make sure that the diameter of the gracilis artery is documented at the desired time periods (for example at 10, 30 and 60 min) of the start of the hyperinsulinemic-euglycemic clamp with the computer program.

9. After $25 \mathrm{~min}$ and/or $55 \mathrm{~min}$ of the insulin clamp, start the second (hyperinsulinemic) CEUS measurement to document the MBV at 30 and/or $60 \mathrm{~min}$, respectively. Follow the same steps described in 2.4 and 2.5. Detach and use the anesthesia port of the 4-way connector to infuse the microbubbles. Reattach the anesthesia tube after the end of the microbubbles infusion.

10. After completion of IVM and the CEUS measurements $60 \mathrm{~min}$ after the start of insulin infusion, withdraw blood from the mouse by a heart puncture procedure for later analysis. This will also euthanize the mouse. Carefully dissect the gracilis and femoral arteries and store them for further desired experiments (for e.g., Western blots, Immunohistochemistry, ex vivo pressure myography experiments ${ }^{36,37,38}$ ).

\section{Offline Analysis}

NOTE: The analyses of IVM and CEUS measurements should be performed offline by a blinded investigator. CEUS offers the possibility to distinguish the microcirculation from larger vessels by temporarily destructing the microbubbles by high intensity ultrasound waves using the MBD function. The signal (measured in arbitrary units (a.u)) in larger vessels is restored quicker than those in the microcirculation because of the microbubbles speed in the corresponding vessels.

1. Use an offline workstation or the software on the ultrasound machine to do the analyses.

2. Draw a region of interests $(\mathrm{ROI})$ to include the microcirculation. Draw a separate ROI to include the larger femoral vessels (Figure $\mathbf{3 A})$.

3. Duplicate the microcirculation's and larger vessels' ROls for the background, baseline and hyperinsulinemic measurements by using the ROI copy function built in the software.

4. Subtract the intensity signal of the background measurement from the baseline and the hyperinsulinemic measurements.

5. Divide the intensity signal of the microcirculation by the intensity signal of the femoral vessels. Baseline and hyperinsulinemic MBVs can now be compared.

\section{Representative Results}

Glucose infusion rate during the hyperinsulinemic-euglycemic clamp (insulin sensitivity) was $180.21 \pm 19.81 \mu \mathrm{mol} / \mathrm{kg} / \mathrm{min}$. Local application of paraffin oil on the adductor muscle compartment to stabilize the vessel did not change the average baseline diameter of the arteries $(73.6 \pm 29.0$ $\mu \mathrm{m}$ vs. $68.8 \pm 17.9 \mu \mathrm{m} ; p=0.58$ ) but helped reduce the variation the animals tested (Figure $4 \mathrm{~A}$ ). Insulin consistently increased the gracilis artery diameter (by $14.58 \pm 6.2 \%$ at $60 \mathrm{~min} ; \mathrm{N}=9$ ) which was significantly different $(p<0.0001)$ from the diameter change caused by saline infusion $(-6.3 \pm 4.9 \% ; N=6)$. Insulin-induced vasodilation was appreciable after $10 \mathrm{~min}(10.09 \pm 5.1 \% ; p=0.002)$ and reached approximately $95 \%$ of its maximum dilatory capacity after $30 \mathrm{~min}$.

Using CEUS, insulin consistently increased muscle MBV (Figure $5 \mathbf{A})$ by $33.5 \%( \pm 31.04 \%, N=7 ; p=0.0009)$ when compared to saline infusion $(-10.63 \pm 27.87 \%, N=6)$ (Figure 5B). The data presented are the signal intensities of the muscle MBV divided by that in the femoral vessels. This reduces experimental variation between different measurements and between different mice (data not shown). The signal intensity in the femoral vessels corresponds linearly with the concentration of microbubbles in the circulation (Figure $3 \mathrm{C}$ ). Correcting for the femoral vessels' signal theoretically corrects for differences in concentrations of microbubbles used (Figure 3D). Data are presented in this section as mean \pm standard deviation. 
A)

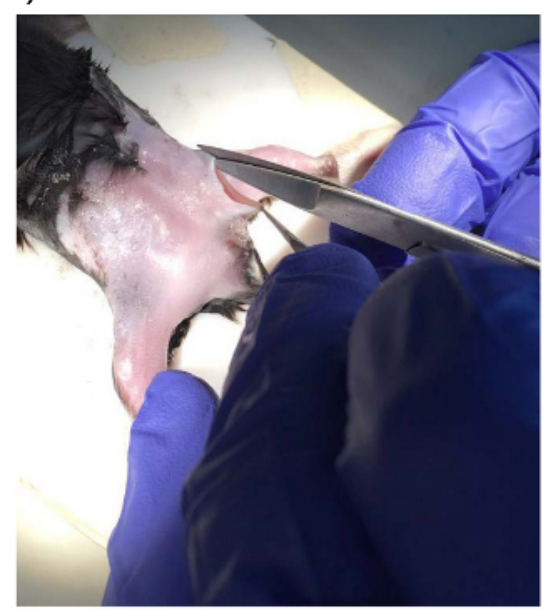

D)

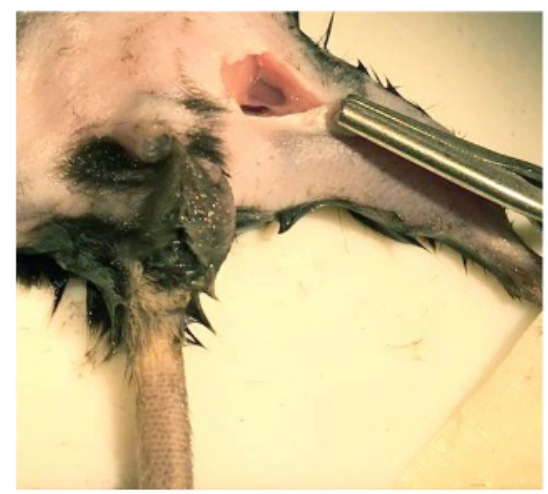

B)

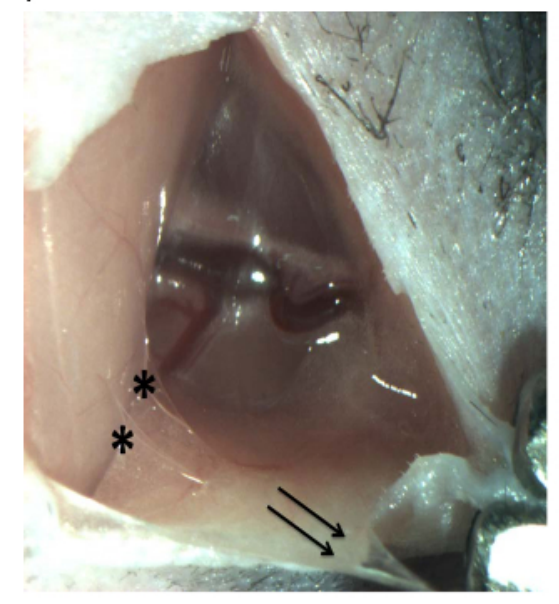

C)

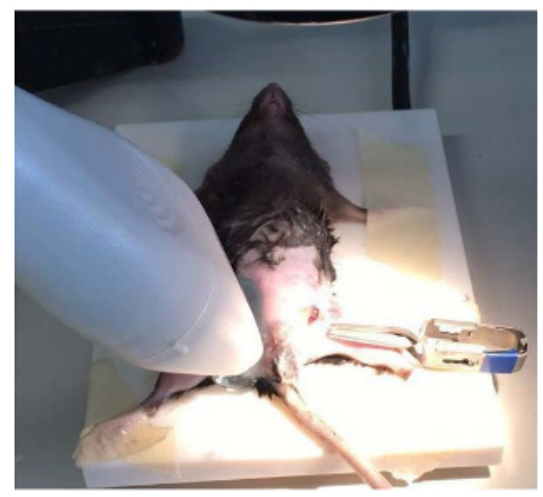

Figure 1: Surgical Exposition of the Adductor Compartment of the Hindlimb. (A) An incision is made at the groin, parallel to the direction of the inguinal ligament. (B) Gentle traction at the fat pad in the distal directions (black arrows) will present the connective tissue $\left({ }^{*}\right)$ between the fat pad and the abdominal wall. (C) The skin folds of the incision can be adjusted using the bulldog clamp to create a small cavity to hold the paraffin oil that bathes the vessels. (D) The ultrasound probe is positioned on the contralateral upper hindlimb after the prepared gracilis artery is viewed using a calibrated microscope. Please click here to view a larger version of this figure. 


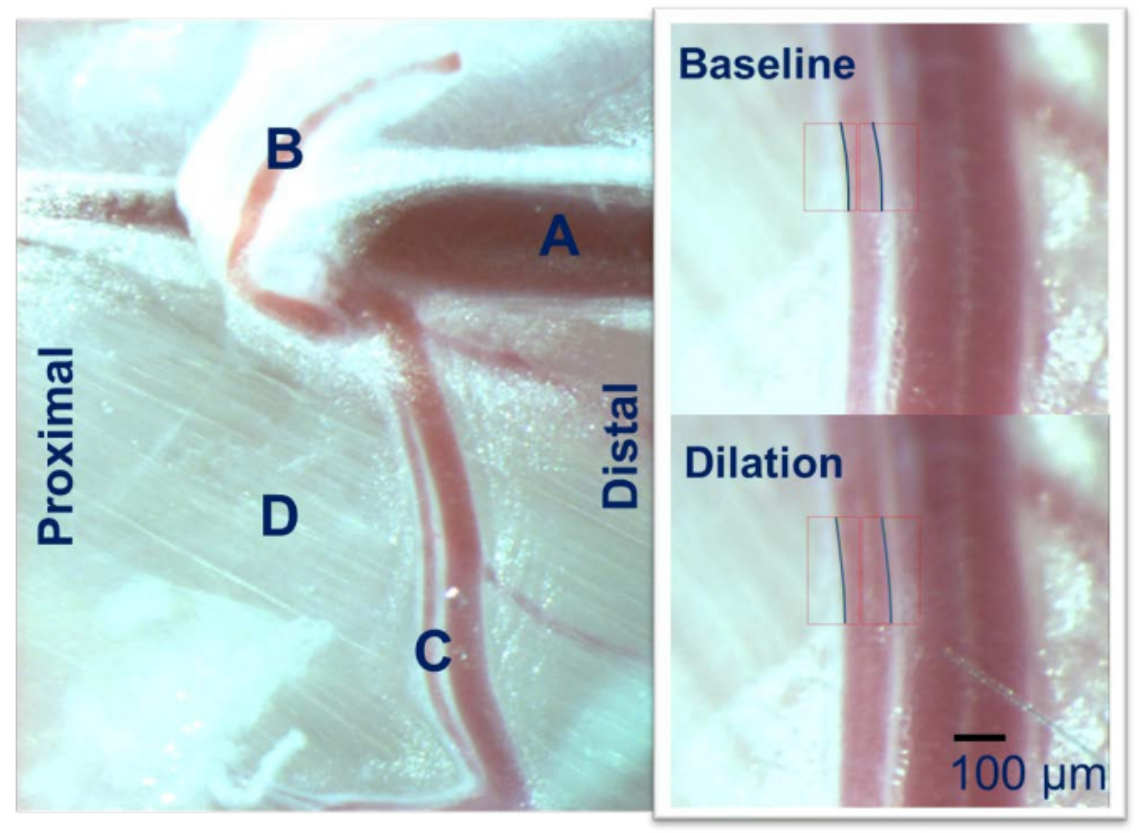

Figure 2: Intravital Microscopy of the Mouse Hindlimb. The femoral artery (A) gives rise to the epigastric artery (B) and the gracilis artery (C) which runs over the adductor muscle group (D). The gracilis artery is used for the IVM using a calibrated microscope. Please click here to view a larger version of this figure. 
A)

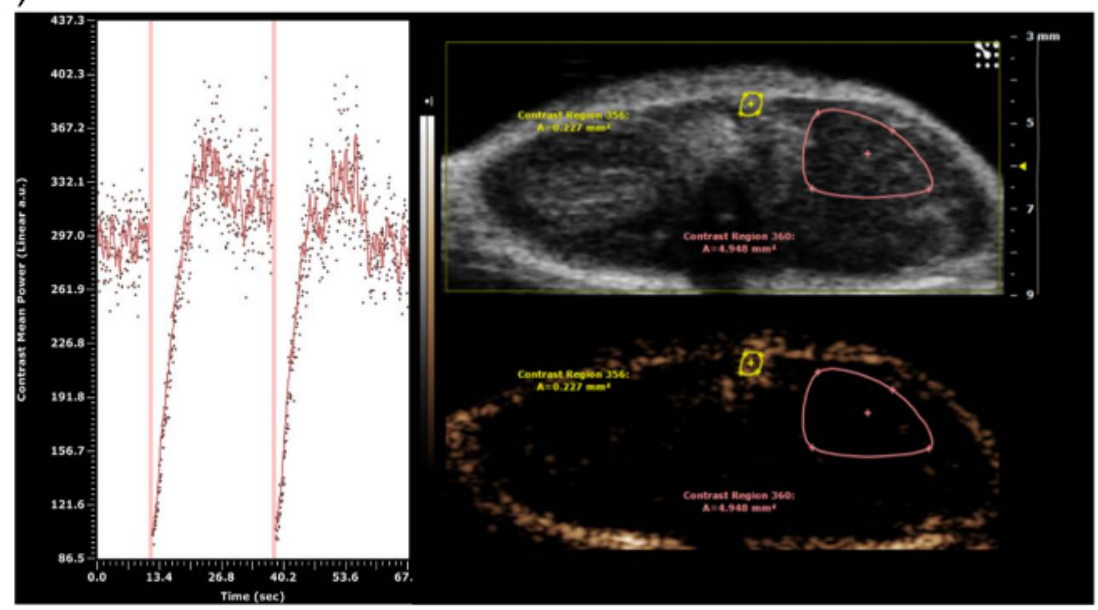

B)

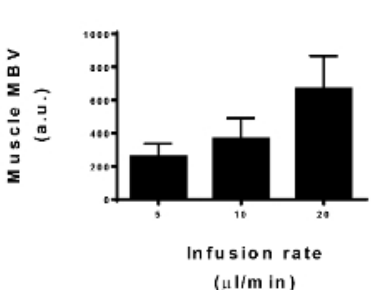

C)

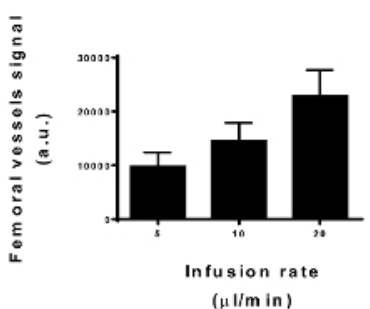

D)

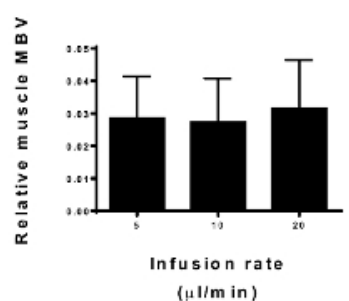

Figure 3: Signal Intensity of Contrast-enhanced Ultrasonography in Muscle Microvascular Blood Volume and Femoral Vessels. (A) View of the nonlinear contrast imaging mode of the digital imaging platform during microbubbles constant infusion in a male mouse upper hindlimb. Right panel: two ROIs are drawn to represent the muscle MBV and the femoral vessels. Only the superficial part of the adductor muscle compartment is included in the ROI as the signal intensity decreases with depth. Left panel: time-intensity curve from the muscle MBV ROI. Vertical lines represent the destruction of the microbubbles (MBD) with high energy waves. Immediately after the MBD, no contrast agent is present in the imaging plane which starts to fill with microbubbles gradually. After 10-15 s, the peak of the contrast enhancement has been reached. (B-D) After a steady-state signal was reached, the infusion rate of $2.5 \times 10^{9}$ bubbles $/ \mathrm{mL}$ was doubled $(5,10,20 \mu \mathrm{L} / \mathrm{min})$. Signal intensity from muscle MBV (B) and femoral vessels (C) paralleled the doubling of the microbubble concentration in the circulation. (D) Correcting muscle MBV for the femoral vessels signal removes the variability in signal intensity caused by different microbubble concentrations $(\mathrm{N}=9$; error-bars represent standard deviation). Please click here to view a larger version of this figure. 
A)

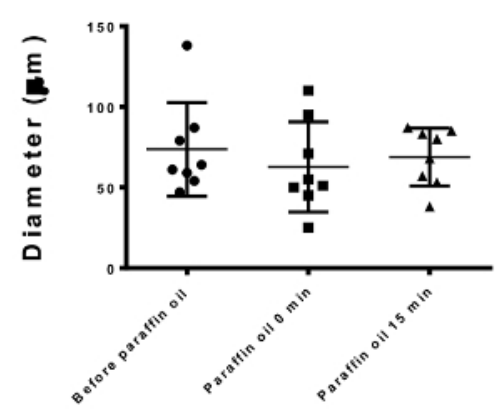

C)

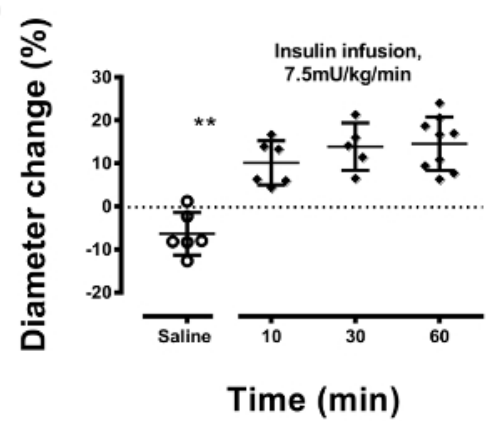

B)

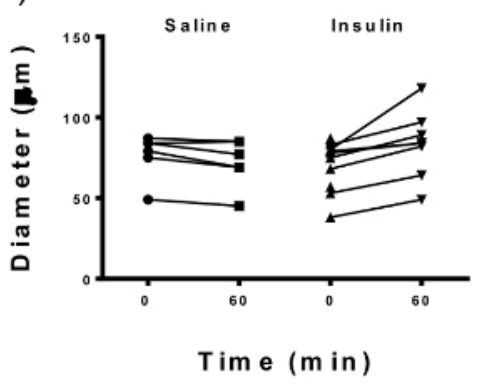

Figure 4: Intravital Microscopy Measurements of the Gracilis Artery. (A) Paraffin oil reduces the variation of the gracilis arteries of different animals (which is $29.0 \mu \mathrm{m}$ without paraffin vs $17.9 \mu \mathrm{m}$ after applying the oil) while keeping the average baseline diameter stable $(73.6 \mu \mathrm{m}$ vs. $68.8 \mu \mathrm{m} ; p=0.58$ ). (B) Arterial diameters at baseline and after $60 \mathrm{~min}$ of insulin or saline infusions. Insulin after 60 min infusion consistently dilated the gracilis artery $(p<0.0001)$ when compared to saline infusion. (C) Insulin-induced vasodilation occurs at 10 min after the start of infusion $(p=0.002)$ and reaches $95 \%$ of the maximum at $30 \mathrm{~min}$. Error-bars represent standard deviation; unpaired Student's T-test is used for statistics. Please click here to view a larger version of this figure.

A)

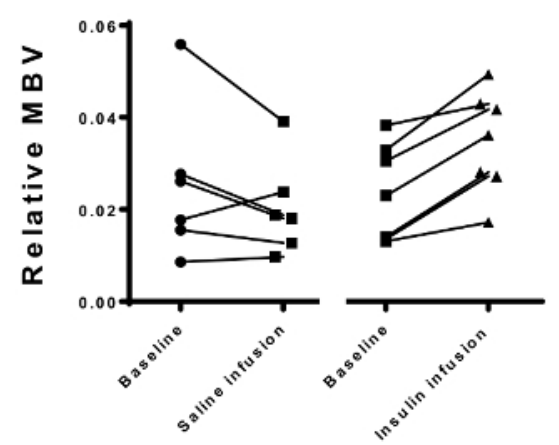

B)

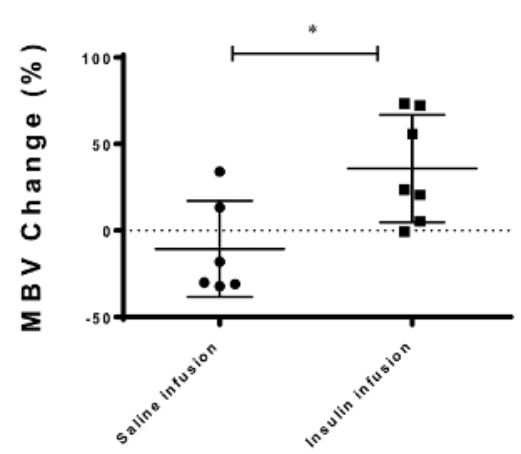

Figure 5: Microvascular Blood Volume Measurements using Contrast-enhanced Ultrasonography of the Adductor Muscle

Compartment of the Mouse Hindlimb. (A) Insulin resulted in a consistent increase in MBV 30 min after the start of insulin infusion. (B) The difference between the hyperinsulinemic and the baseline measurements (MBV change) is denoted as the insulin-mediated microvascular recruitment. Insulin induced a $33.5 \%( \pm 31.04 \%, p=0.016 ; \mathrm{N}=7)$ microvascular recruitment compared to saline infusion $(-10.63 \pm 27.87 \%, \mathrm{~N}$ $=6$ ). Error-bars represent standard deviation; unpaired Student's T-test is used for statistics. Please click here to view a larger version of this figure.

\section{Discussion}

We have developed a technique to simultaneously estimate insulin's vascular actions on the larger arteries (using IVM) and the skeletal muscle microcirculation (using CEUS). The critical steps for successful and reliable measurements are: 1) correctly exposing the gracilis artery without bleeding; 2) preventing leakage of the paraffin oil bathing the artery; 3 ) having a patent venous access (tail vein cannula) for infusion of vasoactive compounds (insulin) and contrast agent (microbubbles). 
The study of microvascular dysfunction in muscle has been gaining attention in the context of obesity and insulin resistance ${ }^{14,25,39,40}$. The negative impact of obesity and insulin resistance on vascular function is manifested at different levels of the vascular tree. Henceforth, different approaches are required to assess these changes. The combined use of the IVM and CEUS techniques in the same mouse provides a powerful tool to quantify insulin's effects at different levels of the vasculature. IVM allows direct visualization and quantitative analysis of the resistance artery and CEUS allows for assessment of insulin-induced changes in muscle perfusion.

Studying the adductor muscle compartment has several advantages. The arteries are readily accessible and the superficial nature of the incision makes it possible to close the skin incision with a 5.0 absorbable sterile suture after the experiment is finished. The animals were injected subcutaneously with buprenorphine after the experiments as an analgesic at a dose of $0.1 \mathrm{mg} / \mathrm{kg}$ and allowed to recover in a warm environment. The mice tolerated the procedure very well and we experienced no loss of animals nor infections of the hindlimb in more than 35 animals studied. This makes it possible to follow-up or study the animals in a longitudinal fashion. The animals used in these experiments, however, were anesthetized using $1.8 \%$ inhaled isoflurane balanced with oxygen flowing at $0.4 \mathrm{~L} / \mathrm{min}$ though an anesthesia mask. In contrast to isoflurane anesthesia $^{41,42}$, FMA anesthesia does not disturb peripheral insulin sensitivity. A future plan is to study how well the mice recover from FMA anesthesia.

The adductor muscle compartment is also useful since various vasoactive compounds mediating local and downstream vascular effects can be evaluated. For example, topical application of these compounds to the target tissue is feasible using superfusion techniques ${ }^{28}$ or surgical manipulation and implantation of drug-eluting cuffs surrounding the vessels ${ }^{43}$. Furthermore, the gracilis artery can be isolated and studied in the pressure myograph. Our group and others have gathered substantial experimental evidence using the pressure myograph to document the effects of insulin and other vasoactive compounds on this artery ex vivo ${ }^{36,37,38}$.

A limitation inherent to the use of the IVM technique is the surgical exposition of the muscle and application of paraffin oil to stabilize the vessels. It is not clear whether these actions impact the native environment of the artery. However, Figure 3A shows that the baseline diameter of the gracilis artery bathed in paraffin oil does not change considerably. It has been also shown that mineral oil successfully inhibits the diffusion of oxygen, protecting the tissue from hyperoxic conditions ${ }^{44}$. Moreover, the oil helps to reduce the variation in the baseline diameter of the arteries. This is why we advocate to use paraffin oil and let the preparation rest for at least $30 \mathrm{~min}$. Of note, the use of buffered saline instead of oil - or no oil at all - resulted in highly variable diameters and constriction of the vessel (data not shown). Moreover, at the end of the experiments, we isolated the gracilis arteries - bathed in paraffin oil - and tested their reactivity in the pressure myograph ex vivo. The paraffin oil-bathed arteries reacted similarly to control arteries when stimulated with insulin and acetylcholine (a vasodilator) (data not shown). The consistent insulininduced vasodilation clearly shows that the IVM protocol described in this study produces reliable results.

The advantage of applying both techniques in the same mouse overcomes some of the intrinsic limitations of one technique by the other: CEUS estimates MBV in the undisturbed muscle in vivo, but individual vessels cannot be seen; IVM makes it possible to see individual vessels, albeit it cannot estimate MBV. A future plan is to utilize IVM microscopy of the cremaster muscle in combination with CEUS of the adductor muscle on the contralateral side. This modification can provide an estimate of the MBV (using CEUS) and a direct optical access to the capillaries (using IVM). The protocol can be further modified; the 4-way connector used for the tail cannula can be switched to a 5-way connector. By this, we can avoid detaching the anesthesia tube while performing the second CEUS measurement (described in point 2.9). In our experience, the mice tolerated the current protocol well. Another modification that can be made to this protocol is the insulin clamp rate used. We used $7.5 \mathrm{mU} / \mathrm{kg} / \mathrm{min}$ clamp rate which is considered supra-physiological. Depending on the study, a lower insulin clamp rate (for example $3 \mathrm{mU} / \mathrm{kg} / \mathrm{min}$ ) can be used.

While we have found the described protocol reliable, there are specific limitations that need attention. There are situations when the measurement of the arterial diameter is not optimal. Executing the preparation steps requires some experience with the model. It is crucial that the paraffin oil does not leak from the vessel environment as supplementing it with new oil will disturb the vessel and change the diameter, making it necessary to let the artery rest for another $30 \mathrm{~min}$. In addition, the reflection of the light (described in step 1.14 of the protocol) on the surface of the paraffin oil was sometimes too bright, making it difficult to view the artery. This can be counteracted by directing the light source so that the light falls at an angle to the paraffin oil surface and parallel to the artery.

In conclusion, the combination of IVM and CEUS techniques described in this study makes it possible to quantify different effects of insulin at different levels of the vasculature. IVM of the gracilis artery provides insight into the upstream vascular changes contributing to downstream microvascular perfusion measured using CEUS. We advocate the combination of several experimental techniques in the same mouse to better assess the vascular function.

\section{Disclosures}

Visual Sonics Inc. covered the open access fees, whereas the structure and content of the article remained the full responsibility of the authors.

\section{Acknowledgements}

We thank Ing. Duncan van Groen for programming the image analysis software (ImageGrabber) used in this study. Funding for this research has been provided by a VIDI grant from the Netherlands Organisation for Scientific Research (grant 016.136.372).

\section{References}

1. DeFronzo, R. A., Tobin, J. D., Rowe, J. W., \& Andres, R. Glucose intolerance in uremia. Quantification of pancreatic beta cell sensitivity to glucose and tissue sensitivity to insulin. The J Clin Invest. 62, 425-435 (1978).

2. Chiu, J. D. et al. Direct administration of insulin into skeletal muscle reveals that the transport of insulin across the capillary endothelium limits the time course of insulin to activate glucose disposal. Diabetes. 57, 828-835 (2008).

3. Herkner, H. et al. Transcapillary insulin transfer in human skeletal muscle. Eur J Clin Invest. 33, 141-146 (2003). 
4. Yang, Y. J., Hope, I. D., Ader, M., \& Bergman, R. N. Insulin transport across capillaries is rate limiting for insulin action in dogs. J Clin Invest. 84, 1620-1628 (1989).

5. Clerk, L. H. et al. The vasodilatory actions of insulin on resistance and terminal arterioles and their impact on muscle glucose uptake. Diabetes Metab Res Rev. 20, 3-12 (2004).

6. Vincent, M. A. et al. Microvascular recruitment is an early insulin effect that regulates skeletal muscle glucose uptake in vivo. Diabetes. $\mathbf{5 3}$ 1418-1423 (2004).

7. Barrett, E. J. et al. The vascular actions of insulin control its delivery to muscle and regulate the rate-limiting step in skeletal muscle insulin action. Diabetologia. 52, 752-764 (2009).

8. Vincent, M. A., Clerk, L. H., Rattigan, S., Clark, M. G., \& Barrett, E. J. Active role for the vasculature in the delivery of insulin to skeletal muscle. Clin Exp Pharmacol Physiol. 32, 302-307 (2005).

9. Clark, M. G., Rattigan, S., Barrett, E. J., \& Vincent, M. A. Point: There is capillary recruitment in active skeletal muscle during exercise. J Appl Physiol (1985). 104, 889-891 (2008).

10. Wallis, M. G. et al. Insulin-mediated hemodynamic changes are impaired in muscle of Zucker obese rats. Diabetes. 51, $3492-3498$ (2002).

11. Eringa, E. C., Stehouwer, C. D., Roos, M. H., Westerhof, N., \& Sipkema, P. Selective resistance to vasoactive effects of insulin in muscle resistance arteries of obese Zucker (fa/fa) rats. Am J Physiol Endocrinol Metab. 293, E1134-1139 (2007).

12. Bonner, J. S. et al. Muscle-specific vascular endothelial growth factor deletion induces muscle capillary rarefaction creating muscle insulin resistance. Diabetes. 62, 572-580 (2013).

13. Kubota, T. et al. Impaired insulin signaling in endothelial cells reduces insulin-induced glucose uptake by skeletal muscle. Cell Metab. 13, 294-307 (2011).

14. Levy, B. I. et al. Impaired tissue perfusion: a pathology common to hypertension, obesity, and diabetes mellitus. Circulation. 118, 968-976 (2008).

15. Schelbert, H. R. Coronary circulatory function abnormalities in insulin resistance: insights from positron emission tomography. J Am Coll Cardiol. 53, S3-8 (2009).

16. Wong, T. Y. et al. Associations between the metabolic syndrome and retinal microvascular signs: the Atherosclerosis Risk In Communities study. Invest Ophthalmol Vis Sci. 45, 2949-2954 (2004).

17. Bonadonna, R. C. et al. Role of tissue-specific blood flow and tissue recruitment in insulin-mediated glucose uptake of human skeletal muscle. Circulation. 98, 234-241 (1998).

18. Ellmerer, M. et al. Reduced access to insulin-sensitive tissues in dogs with obesity secondary to increased fat intake. Diabetes. $\mathbf{5 5}$, 1769-1775 (2006)

19. Ellmerer, M. et al. Physiological hyperinsulinemia in dogs augments access of macromolecules to insulin-sensitive tissues. Diabetes. $\mathbf{5 3}$ 2741-2747 (2004).

20. Vincent, M. A. et al. Mixed meal and light exercise each recruit muscle capillaries in healthy humans. Am J Physiol Endocrinol Metab. 290, E1191-1197 (2006).

21. Jongh, R. T., Serne, E. H., RG, I. J., de Vries, G., \& Stehouwer, C. D. Impaired microvascular function in obesity: implications for obesityassociated microangiopathy, hypertension, and insulin resistance. Circulation. 109, 2529-2535 (2004).

22. Ketel, I. J. et al. Obese but not normal-weight women with polycystic ovary syndrome are characterized by metabolic and microvascular insulin resistance. J Clin Endocrinol Metab. 93, 3365-3372 (2008).

23. Khan, F. et al. Impaired microvascular function in normal children: effects of adiposity and poor glucose handling. J Physiol. 551, 705-711 (2003).

24. Clark, M. G. Impaired microvascular perfusion: a consequence of vascular dysfunction and a potential cause of insulin resistance in muscle. Am J Physiol Endocrinol Metab. 295, E732-750 (2008).

25. Serne, E. H. et al. Impaired skin capillary recruitment in essential hypertension is caused by both functional and structural capillary rarefaction. Hypertension. 38, 238-242 (2001).

26. Sriramarao, P., Anderson, W., Wolitzky, B. A., \& Broide, D. H. Mouse bone marrow-derived mast cells roll on P-selectin under conditions of flow in vivo. Lab Invest. 74, 634-643 (1996).

27. Leister, I. et al. A peritoneal cavity chamber for intravital microscopy of the liver under conditions of pneumoperitoneum. Surg Endosc. 17, 939-942 (2003).

28. Cardinal, T. R. et al. Chronic hindlimb ischemia impairs functional vasodilation and vascular reactivity in mouse feed arteries. Front Physiol. 2 , $91(2011)$

29. Duling, B. R. The preparation and use of the hamster cheek pouch for studies of the microcirculation. Microvasc Res. 5, 423-429 (1973).

30. Wei, K. et al. Quantification of myocardial blood flow with ultrasound-induced destruction of microbubbles administered as a constant venous infusion. Circulation. 97, 473-483 (1998).

31. Clerk, L. H., Rattigan, S., \& Clark, M. G. Lipid infusion impairs physiologic insulin-mediated capillary recruitment and muscle glucose uptake in vivo. Diabetes. 51, 1138-1145 (2002).

32. Barrett, E. J., Keske, M. A., Rattigan, S., \& Eringa, E. C. CrossTalk proposal: De novo capillary recruitment in healthy muscle is necessary. $J$ Physiol. 592, 5129-5131 (2014).

33. Aman, J. et al. Effective treatment of edema and endothelial barrier dysfunction with imatinib. Circulation. 126, 2728-2738 (2012).

34. Boer, M. P. et al. Globular adiponectin controls insulin-mediated vasoreactivity in muscle through AMPKalpha2. Vascul Pharmacol. 78, 24-35 (2016).

35. Brom, C. E. et al. Myocardial Perfusion and Function Are Distinctly Altered by Sevoflurane Anesthesia in Diet-Induced Prediabetic Rats. $J$ Diabetes Res. 2016, 5205631 (2016)

36. Meijer, R. I. et al. Perivascular adipose tissue control of insulin-induced vasoreactivity in muscle is impaired in $\mathrm{db} / \mathrm{db}$ mice. Diabetes. 62 , 590-598 (2013).

37. Meijer, R. I. et al. Insulin-induced changes in skeletal muscle microvascular perfusion are dependent upon perivascular adipose tissue in women. Diabetologia. 58, 1907-1915 (2015).

38. Sun, D., Kaley, G., \& Koller, A. Characteristics and origin of myogenic response in isolated gracilis muscle arterioles. Am J Physiol. 266, H1177-1183 (1994).

39. Jonk, A. M. et al. Microvascular dysfunction in obesity: a potential mechanism in the pathogenesis of obesity-associated insulin resistance and hypertension. Physiology (Bethesda). 22, 252-260 (2007). 
40. Wiernsperger, N., Nivoit, P., De Aguiar, L. G., \& Bouskela, E. Microcirculation and the metabolic syndrome. Microcirculation. 14, 403-438 (2007).

41. Horber, F. F. et al. Isoflurane and whole body leucine, glucose, and fatty acid metabolism in dogs. Anesthesiology. 73, 82-92 (1990).

42. Sui, H. et al. Quantifying insulin sensitivity and entero-insular responsiveness to hyper- and hypoglycemia in ferrets. PLoS One. 9, e90519 (2014).

43. Pires, N. M. et al. Local perivascular delivery of anti-restenotic agents from a drug-eluting poly(epsilon-caprolactone) stent cuff. Biomaterials. 26, 5386-5394 (2005)

44. Young, D. A., Chi, M. M., \& Lowry, O. H. Energy metabolism of skeletal muscle biopsies stimulated anaerobically without load in vitro. Am J Physiol. 250, C813-820 (1986). 